\title{
Cadaveric-biomechanical study on medial retinaculum: its stabilising role for the patella against lateral dislocation
}

\author{
L. Mitrogiannis' ${ }^{1}$ A. Barbouti ${ }^{1}$, P. Kanavaros ${ }^{1}$, G. Paraskevas², A. Kitsouli', \\ G. Mitrogiannis ${ }^{1}$, P. Kitsoulis ${ }^{1}$
}

${ }^{1}$ Laboratory of Anatomy-Histology-Embryology, School of Medicine, University of loannina, Greece 'Laboratory of Anatomy-Histology-Embryology, School of Medicine, Aristotle University, Thessaloniki, Greece

[Received: 27 January 2018; Accepted: 8 March 2018]

\begin{abstract}
Background: The aim of this study was to analyse the biomechanical role of medial retinaculum, as a stabilising factor against lateral patellar dislocation.

Materials and methods: This cadaveric-biomechanical study included the patellae of 10 cadaveric knees, which were surgically exposed and the medial retinaculum of each one was located. A stable $24.51 \mathrm{~N}$ force was applied to the four parts of the quadriceps, and an increasing lateral displacing force was applied to the patella, up to $5 \mathrm{~mm}$ dislocation. The study was repeated for $0^{\circ}, 45^{\circ}$, and $90^{\circ}$ of knee flexion, with the medial retinaculum intact and dissected. The Wilcoxon signed rank test was used for data analysis. A p value $<0.05$ was considered as statistical significant.

Results: After the dissection of medial retinaculum, the lateral displacement force was lower at every angle of knee flexion ( $p=0.005, p=0.007, p=0.005$, respectively). The lateral displacement force increased as the flexion angle increased $(p=0.005)$, regardless of medial retinaculum integrity.

Conclusions: Medial retinaculum acts as a stabilising factor for the patella, against its lateral dislocation in lower flexion angles. Therefore, methods of surgical reinforcement or repair of medial retinaculum could provide protection against recurrent patellar dislocation. (Folia Morphol 2018; 77, 4: 742-747)
\end{abstract}

Key words: patellar instability, patellar retinaculum, medial stabilisers, lateral displacement force, medial patellofemoral ligament

\section{INTRODUCTION}

Patellar dislocation is a common occurrence in children and adolescents, characterised by lateral dislocation of the patella, usually followed by spontaneous reduction. During patella dislocation, soft-tissue structures, such as the medial patellofemoral ligament (MPFL), medial retinaculum (MR) and vastus medialis obliquus (VMO) muscle fibres, are usually injured, frequently leading to recurrent dislocations [9].

Dislocation which occurs for the first time is usually treated conservatively, with some exceptions, such as in the presence of osteochondral fragments or important soft tissue damage [7]. Recurrent and chronic dislocations are usually treated by medial structure repair. Most common procedures include reconstruction of the MPFL, reinforcement of the $M R$, with or without lateral release, medialisation of the insertion of the patellar ligament, trochleoplasty, and tibial tubercle-trochlear groove (TT-TG) distance change [7].

As far as the MPFL is concerned, cadaveric studies showed a contribution of up to $50-60 \%$ to the stabil- 
ity of the patella $[5,13]$. Other stabilising structures are the medial patellomenisceal ligament (MPML) and the medial patellotibial ligament (MPTL) [13], plus the $M R$, which was the main subject of our study.

There are many studies in the literature proposing methods of surgical reinforcement of the MR, such as MR reefing [1], plasty [8], imbrication [16], plication [21], thermal shrinkage [2], whether arthroscopic $[3,14,15,18]$ or not. In most studies the outcomes were positive, showing that the MR plays an important role as a stabilising factor for the patella. There have also been studies comparing methods of MR repair and repair of other medial stabilisers, such as the vastus medialis [20] and MPFL [21], showing that MPFL is a better static stabiliser compared to MR. Studies dealing with MPFL repair alone also showed good clinical results, reinforcing the view that MPFL is the main stabiliser against lateral displacement of the patella $[4,5,10,11,12,17,19]$, especially in lower angles of flexion.

The main objective of this cadaveric study was to evaluate the contribution of the MR as a lateral patellar stabiliser for the knee. The study hypothesis was that the MR provides adequate stability against lateral displacement forces, when intact.

According to the above-mentioned literature, surgical interventions reinforcing the MR yield good outcomes in terms of the lateral stability of the patella, in cases of recurrent patella dislocation. These methods can usually be performed arthroscopically, which is quite important regarding the surgical complications. With this cadaveric biomechanical study, we tried to show that an intact MR is important for the stability of the patella, especially against lateral displacement forces, thereby making a contribution to the current literature.

\section{MATERIALS AND METHODS}

The patellae and surrounding soft tissues of 10 frozen cadaveric knees, with no known previous pathology or anatomical variation, were surgically exposed, using an incision across the two poles of the patella, extended proximally and distally by $5 \mathrm{~cm}$. The MPFL and MR were identified and marked using a coloured marker. The centre of the patella was located and marked using a coloured stitch, while an ethibond stitch was attached to the lateral and middle sides of the soft tissue surrounding the patella. Finally, four ethibond stitches were attached and clothed around the mass of the four parts of the

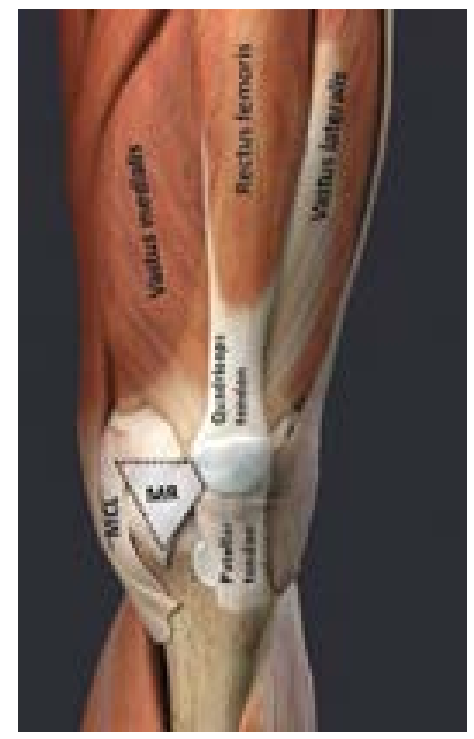

Figure 1. The quadriceps parts and medial structures of the knee: rectus femoris, vastus medialis, vastus lateralis, vastus intermedius (deeper to rectus femoris), medial collateral ligament (MCL), medial retinaculum (MR).

quadriceps muscle (rectus femoris, vastus medialis, vastus lateralis, vastus intermedius; Fig. 1).

Each knee was then placed on the study table, with the lateral side of the patella facing upwards. So, it was possible to flex and extend the knee and the degrees of flexion could be monitored using a goniometer, on the table.

The other end of the stitch attached to the lateral-middle side of the patella, was then rolled over a rod above and parallel to the table surface, and was finally attached to a dynamometer. The ends of the four stitches attached to the quadriceps muscle were attached to weights, hanging out and below the study table (Fig. 2). So, a $24.51 \mathrm{~N}$ ( $2.5 \mathrm{~kg}$ ) force was constantly applied to the patella through the quadriceps muscle and tendon, partially simulating the stabilising forces applied to the patella by quadriceps in vivo. Finally, a numbered ruler was placed next to the marked patella centre, for measuring the patella's lateral displacement.

In this study table setup, we measured the forces that displaced the patella laterally by $5 \mathrm{~mm}$ (lateral displacement force), in six distinct cases: with the MR intact with knee flexed to $0^{\circ}, 45^{\circ}$ and $90^{\circ}$ and with the MR dissected with knee flexed to $0^{\circ}, 45^{\circ}$ and $90^{\circ}$.

\section{Statistical analysis}

The data collected was statistically analysed using the non-parametric Wilcoxon signed rank test (SPSS, version 23, Chicago, USA for OsX), due to paired data 


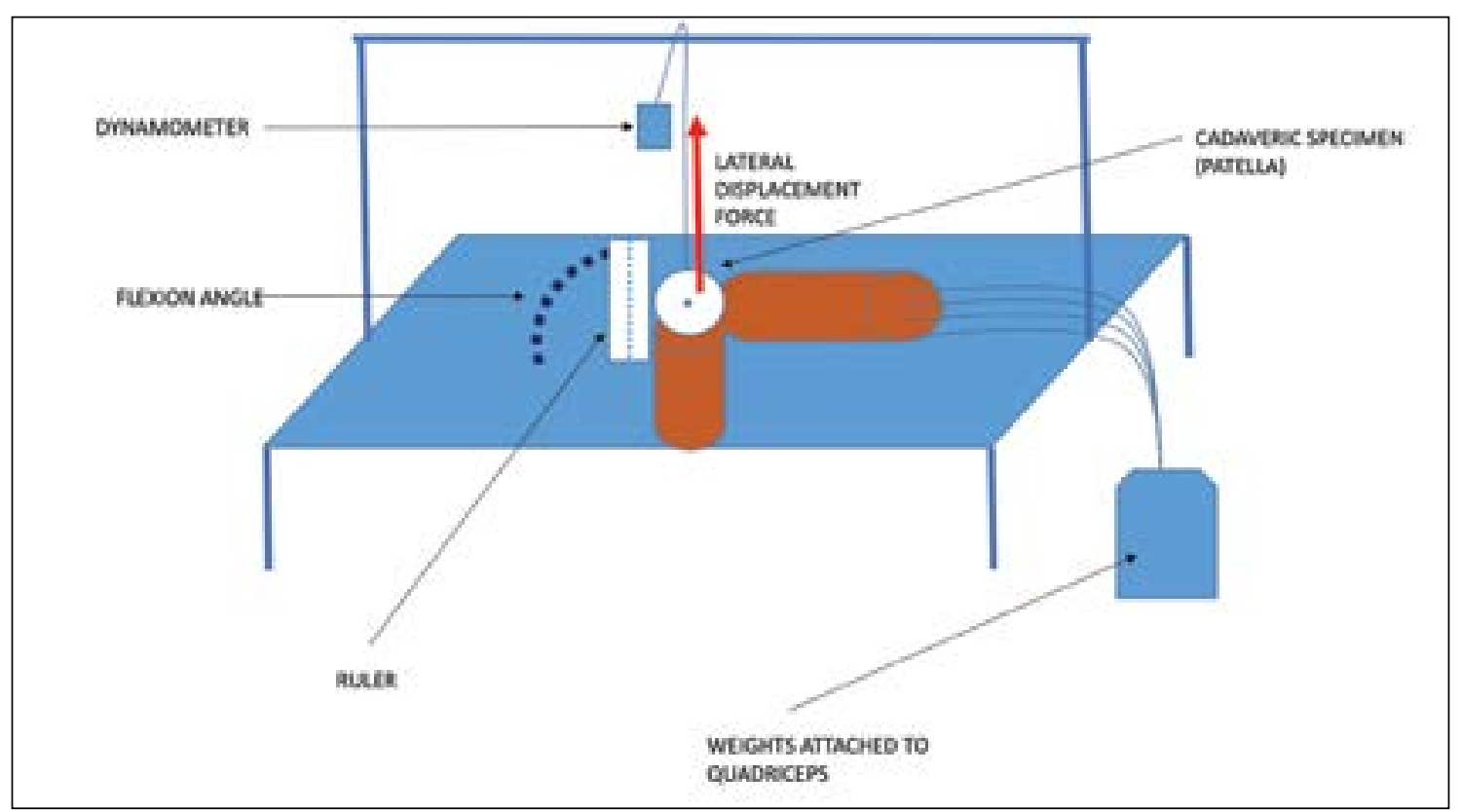

Figure 2. Study table setup.

Table 1. Measurement results

\begin{tabular}{|c|c|c|c|c|c|c|}
\hline & $\begin{array}{c}\text { Intact-extension } \\
{[N]}\end{array}$ & $\begin{array}{c}\text { Intact-45 flexion } \\
{[N]}\end{array}$ & $\begin{array}{c}\text { Intact-90 flexion } \\
{[\mathrm{N}]}\end{array}$ & $\begin{array}{l}\text { Dissected-extension } \\
{[\mathrm{N}]}\end{array}$ & $\begin{array}{l}\text { Dissected-45 extension } \\
{[\mathrm{N}]}\end{array}$ & $\begin{array}{l}\text { Dissected-90 extension } \\
{[\mathrm{N}]}\end{array}$ \\
\hline Knee no. 1 & 4.13 & 6.99 & 13.18 & 3.04 & 6.36 & 8.92 \\
\hline Knee no. 2 & 3.55 & 4.43 & 9.27 & 2.62 & 3.55 & 9.02 \\
\hline Knee no. 3 & 3.13 & 3.99 & 10.80 & 1.87 & 3.39 & 8.88 \\
\hline Knee no. 4 & 1.67 & 4.41 & 7.93 & 1.01 & 2.78 & 7.89 \\
\hline Knee no. 5 & 2.18 & 3.90 & 9.42 & 1.20 & 2.94 & 8.71 \\
\hline Knee no. 6 & 3.14 & 4.24 & 9.83 & 2.14 & 4.68 & 9.12 \\
\hline Knee no. 7 & 4.22 & 6.02 & 9.89 & 3.06 & 5.42 & 9.11 \\
\hline Knee no. 8 & 4.16 & 6.34 & 9.04 & 3.20 & 5.38 & 8.70 \\
\hline Knee no. 9 & 2.67 & 4.01 & 8.99 & 1.39 & 3.01 & 8.10 \\
\hline Knee no. 10 & 3.02 & 4.20 & 8.90 & 1.70 & 3.23 & 8.12 \\
\hline
\end{tabular}

and small sample size. The Kolmogorov-Smirnov test of normality was also used to ensure whether the data were normally distributed. A p value $<0.05$ was considered as statistical significant. The confidence interval was $95 \%$.

\section{Ethical approval}

This study was conducted after approval by the Medical Research Ethics Committee of the Faculty of Medicine, University of Ioannina, Ioannina, Greece.

\section{RESULTS}

As shown in Table 1 and Figure 3, after statistical analysis of the data, the lateral displacement force applied to the patella was lower after the dissection of the MR at $0^{\circ}, 45^{\circ}$ and $90^{\circ}$ of flexion. In detail, at $0^{\circ}$ the $p$ value was 0.005 , at $45^{\circ} p$ value was 0.007 and finally at $90^{\circ} \mathrm{p}$ value was 0.005 .

We also noticed that the lateral displacement force was higher with increasing flexion angles for the knee ( $p=0.005)$, regardless of MR integrity.

The above-mentioned $p$ values, being statistically significant, reinforced our hypothesis that an intact MR provides adequate stability against lateral displacing forces to the patella, especially at lower angles of flexion of the knee.

\section{DISCUSSION}

The statistical verification of our hypothesis leads to the conclusion that the MR plays an important role 




Figure 3. Data graph (mean with standard error); Comparison between knees: 1) Intact retinacullum — Knee extended to dissected retinacullum - Knee extended, $P$ value $=0.005 ; 2$ ) Intact retinacullum - Knee 45 degrees flexion to dissected retinacullum - Knee 45 degrees flexion, $\mathrm{P}$ value $=0.007 ; 3$ ) Intact retinacullum - Knee 90 degrees flexion to dissected retinacullum - Knee 90 degrees flexion, $P$ value $=0.005$

as a medial stabiliser of the patella, especially in low angles of flexion, since from $20^{\circ}$ to $90^{\circ}$ of knee flexion, the interaction of bones is an additional contribution to the stability of the patella, which is in concordance with the literature $[6,13]$. The MPFL and MPML are major stabilisers, contributing up to $50-60 \%$ and $24 \%$, respectively, while MR and MPTL contributes up to $13 \%[5,13]$. VMO also plays an important role, especially in the first $20-30^{\circ}$ of flexion, due to the meshing of its fibres to MPFL, reinforcing MPFLs stabilising role [13].

Warren and Marshall in 1979 [17] described the anatomy of the medial stabilisers of the knee in detail, documenting three layers of soft tissue. The main components of the medial side of the knee are the MPFL, MPML, MPTL and MR (Figs. 4-6). MPFL originates from the medial femoral epicondyle and inserts to the medial side of the patella, between layers I and II. Its fibres mesh with MR fibres. MPML originates from medial capsulo-meniscal region and inserts to the lower patellar pole. MPTL originates from the medial side of the tibia, $1.5-2 \mathrm{~cm}$ below the joint and $1.5-2 \mathrm{~cm}$ medial to the patellar tendon and inserts to the lower pole of the patella. Its fibres mesh with MR fibres as well. MR fibres come from the deep or crural fascia (layer I: the first facial plane found after a skin incision), the vastus medialis tendon and the quadriceps. MR can be identified in front of the medial collateral ligament (MCL) and meets the patellar tendon. It constitutes layers I and II by Warren and Marshall [13, 17].

Other anatomic factors contributing to the stability of the patella are the interaction of the bones, such

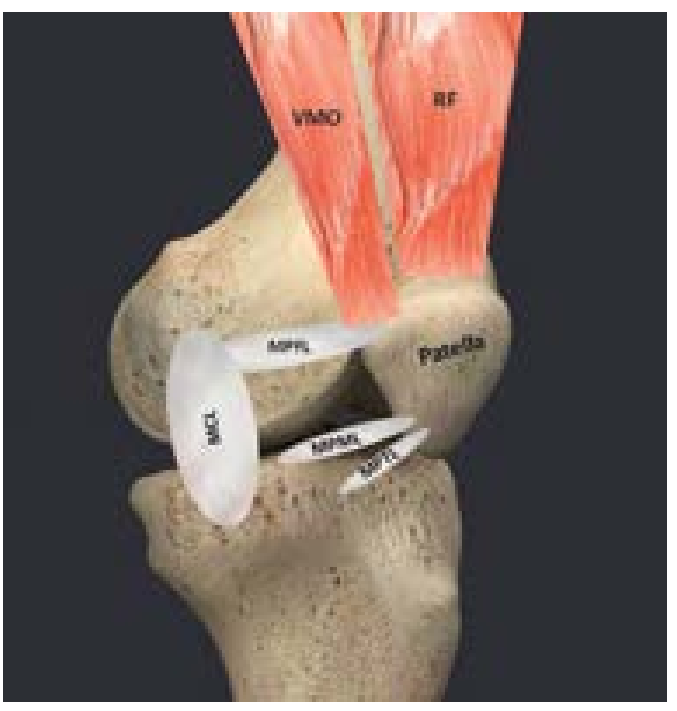

Figure 4. Medial patellofemoral ligament (MPFL), medial patellomenisceal ligament (MPML), medial patellotibial ligament (MPTL), medial retinaculum (MR), vastus medialis obliquus (VMO), rectus femoris (RF), medial collateral ligament (MCL).

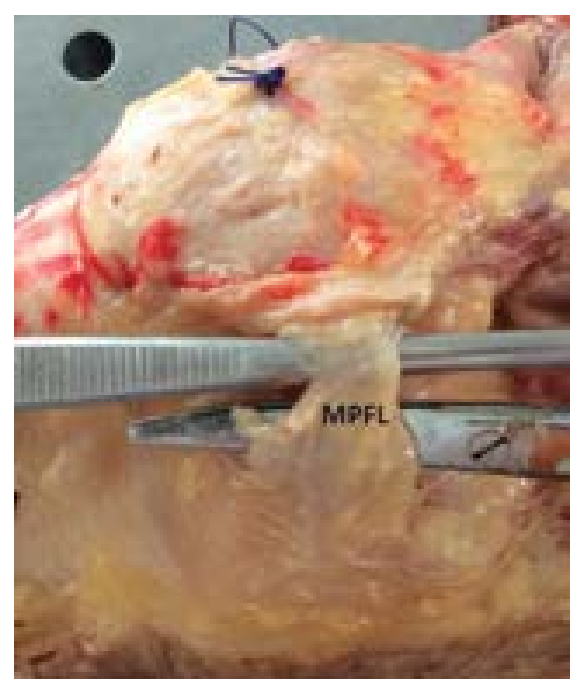

Figure 5. Medial patellofemoral ligament (MPFL) (cadaver).



Figure 6. Medial retinaculum (cadaver). 
as the patella and the trochlear groove, especially in flexed knees (after $20^{\circ}$ ), which changes in cases of a shallow groove or patella alta and baja. The interaction of the quadriceps muscle and the patella, through the force applied to the patella by the muscle (Q-angle), is also an important factor for the stability of the patella.

On the basis of the above, we used ten cadaveric knees with no known anatomical variations or previous pathology. Our anatomic dissection findings were in concordance with the literature $[13,17]$. Despite this, we should note some experimental limitations: the stabilising force applied by the quadriceps muscle to the patella is greater in vivo than in our study, due to the tearing of the cadaver muscle fibres, when force was applied to the muscle, by the ethibond stitches. In vivo, the surrounding tissues apply additional force to the patella, when a human body is in a standing position or in several other states, such as running and jumping. So, the contribution of bone to bone contact and muscle to the stability of the patella is not comparable to in vivo cases.

Despite these limitations, this study quantified the lateral displacement force to the patella, preserving and dissecting the MR and supported the hypothesis that MR plays an important role as a stabiliser against patella lateral dislocation.

\section{CONCLUSIONS}

Although MPFL is the main stabiliser against lateral patellar dislocation and the most common surgical interventions consist of repair or reinforcement of this structure, surgical methods to reinforce the MR, combined or not with methods of relaxation of the lateral soft tissues and anatomical modifications, are important for the treatment of recurrent patella dislocations. So despite the fact that further studies should be conducted on medial patella stabilisers and especially MR, we believe that the surgeons should put in their plan the repair or reinforcement of this structure.

\section{Acknowledgements}

We are grateful to Ms. Stavroula Petridi, Msc., $\mathrm{PhD}$, Biologist/Geneticist for the statistical analysis and interpretation of the results and to loannis Mitrogiannis, undergraduate medical student, who contributed to the laboratory study.

\section{REFERENCES}

1. Cerciello S, Lustig S, Costanzo G, et al. Medial retinaculum reefing for the treatment for patellar instability. Knee Surg Sports Traumatol Arthrosc. 2014; 22(10):
2505-2512, doi: 10.1007/s00167-014-3171-6, indexed in Pubmed: 25059335.

2. Coons DA, Barber FA. Thermal medial retinaculum shrinkage and lateral release for the treatment of recurrent patellar instability. Arthroscopy. 2006; 22(2): 166-171, doi: 10.1016/j. arthro.2005.08.043, indexed in Pubmed: 16458802.

3. Dainer RD, Barrack RL, Buckley SL, et al. Arthroscopic treatment of acute patellar dislocations. Arthroscopy. 1988; 4(4): 267-271, indexed in Pubmed: 3233116.

4. Duchman KR, DeVries NA, McCarthy MA, et al. Biomechanical evaluation of medial patellofemoral ligament reconstruction. lowa Orthop J. 2013; 33: 64-69, indexed in Pubmed: 24027463.

5. Ebied AM, El-Kholy W. Reconstruction of the medial patello-femoral and patello-tibial ligaments for treatment of patellar instability. Knee Surg Sports Traumatol Arthrosc. 2012; 20(5): 926-932, doi: 10.1007/s00167-011-1659-x, indexed in Pubmed: 21935619.

6. Farahmand F, Naghi Tahmasbi M, Amis A. The contribution of the medial retinaculum and quadriceps muscles to patellar lateral stability--an in-vitro study. Knee. 2004; 11(2): 89-94, doi: 10.1016/j.knee.2003.10.004, indexed in Pubmed: 15066616.

7. Frosch S, Balcarek P, Walde TA, et al. [The treatment of patellar dislocation: a systematic review]. Z Orthop Unfall. 2011; 149(6): 630-645, doi: 10.1055/s-0030-1250691, indexed in Pubmed: 21544786.

8. Ma LF, Wang F, Chen BC, et al. Medial retinaculum plasty versus medial patellofemoral ligament reconstruction for recurrent patellar instability in adults: a randomized controlled trial. Arthroscopy. 2013; 29(5): 891-897, doi: 10.1016/j. arthro.2013.01.030, indexed in Pubmed: 23566568.

9. Meyers AB, Laor T, Sharafinski M, et al. Imaging assessment of patellar instability and its treatment in children and adolescents. Pediatr Radiol. 2016; 46(5): 618-636, doi: 10.1007/ s00247-015-3520-8, indexed in Pubmed: 26860094.

10. Mikashima Y, Kimura M, Kobayashi Y, et al. Clinical results of isolated reconstruction of the medial patellofemoral ligament for recurrent dislocation and subluxation of the patella. Acta Orthop Belg. 2006; 72(1): 65-71, indexed in Pubmed: 16570897.

11. Neri T, Philippot R, Carnesecchi O, et al. Medial patellofemoral ligament reconstruction: clinical and radiographic results in a series of 90 cases. Orthop Traumatol Surg Res. 2015; 101(1): 65-69, doi: 10.1016/j.otsr.2014.09.023, indexed in Pubmed: 25530480.

12. Ostermeier S, Stukenborg-Colsman C, Hurschler C, et al. In vitro investigation of the effect of medial patellofemoral ligament reconstruction and medial tibial tuberosity transfer on lateral patellar stability. Arthroscopy. 2006; 22(3): 308-319, doi: 10.1016/j.arthro.2005.09.024, indexed in Pubmed: 16517316.

13. Panagiotopoulos E, Strzelczyk P, Herrmann M, et al. Cadaveric study on static medial patellar stabilizers: the dynamizing role of the vastus medialis obliquus on medial patellofemoral ligament. Knee Surg Sports Traumatol Arthrosc. 2006; 14(1): 7-12, doi: 10.1007/s00167-0050631-z, indexed in Pubmed: 16001289.

14. Rillmann P, Fischer A, Berbig R, et al. [Arthroscopic repair of the medial retinaculum after first time dislocation of the patella]. Unfallchirurg. 1999; 102(3): 167-172, indexed in Pubmed: 10232032. 
15. Schöttle PB, Scheffler SU, Schwarck A, et al. Arthroscopic medial retinacular repair after patellar dislocation with and without underlying trochlear dysplasia: a preliminary report. Arthroscopy. 2006; 22(11): 1192-1198, doi: 10.1016/j.arthro.2006.07.002, indexed in Pubmed: 17084296.

16. Shelbourne KD, Urch SE, Gray T. Results of medial retinacular imbrication in patients with unilateral patellar dislocation. J Knee Surg. 2012; 25(5): 391-396, doi: 10.1055/s0032-1313750, indexed in Pubmed: 23150348.

17. Warren LF, Marshall JL. The supporting structures and layers on the medial side of the knee: an anatomical analysis. J Bone Joint Surg Am. 1979; 61(1): 56-62, indexed in Pubmed: 759437.

18. Yamamoto RK. Arthroscopic repair of the medial retinaculum and capsule in acute patellar dislocations. Arthroscopy. 1986; 2(2): 125-131, indexed in Pubmed: 3730064.
19. Zaffagnini S, Colle F, Lopomo N, et al. The influence of medial patellofemoral ligament on patellofemoral joint kinematics and patellar stability. Knee Surg Sports Traumatol Arthrosc. 2013; 21(9): 2164-2171, doi: 10.1007/ s00167-012-2307-9, indexed in Pubmed: 23179455.

20. Zhao J, Huangfu X, He Y, et al. Recurrent patellar dislocation in adolescents: medial retinaculum plication versus vastus medialis plasty. Am J Sports Med. 2012; 40(1): 123-132, doi: 10.1177/0363546511420551, indexed in Pubmed: 21900625.

21. Zhao J, Huangfu $X, \mathrm{He} Y$. The role of medial retinaculum plication versus medial patellofemoral ligament reconstruction in combined procedures for recurrent patellar instability in adults. Am J Sports Med. 2012; 40(6): 1355-1364, doi: 10.1177/0363546512439193, indexed in Pubmed: 22437282. 